\title{
Community Residents' Awareness of Family Doctor Contract Services, Signing With Family Doctor, Willingness to Use Community Health Care Centers: A Community-Based Analysis in Shenzhen, China
}

\section{Qingming Zheng}

Shenzhen Luohu Disease Prevention and Control Center

Lu Shi ( $\nabla$ shil@oregonstate.edu )

Oregon State University

Tiantian Pang

University of South Florida

Willie Leung

Oregon State University

\section{Research Article}

Keywords: family doctor contract services, family doctor, Andersen's Behavioral Model, Shenzhen, awareness, chronic disease, community health care center

Posted Date: December 14th, 2020

DOI: https://doi.org/10.21203/rs.3.rs-118080/v1

License: @ (i) This work is licensed under a Creative Commons Attribution 4.0 International License. Read Full License 


\section{Abstract}

Background: Family doctor contracted services (FDCS) began in China in 2016. Shenzhen, one of the most developed cities in China, implemented a family doctor (FD) policy in 2017. The objectives of this study were to identify the impact of the awareness of the FD and signing with a FD on health care seeking behavior, as well as the impact of chronic disease status on the awareness of FDs and signing with a FD.

Methods: Cross-sectional secondary data based on residents living in Luohu district was used for analysis. Linear probability models (LPM) were used to determine the relationship between willingness to use community health care centers (CHCs) and awareness of the FDCS, as well as the association between willingness to use $\mathrm{CHCs}$ and signing with a FD. LPM was also used to estimate the association between chronic disease status and awareness of the FDCS, as well as the association between chronic disease status and signing with a FD.

Results: Among 1205 adults included in analysis, $27 \%$ of the participants knew the FDCS, $5 \%$ signed with FD, and $20 \%$ had chronic disease. Both awareness of the FDCS and signing with a FD significantly increased the probability of using $\mathrm{CHCs}$. The results indicated that participants with chronic disease were more likely to be aware of the FDCS and sign with a FD.

Conclusions: Consequently, this study provided evidence that awareness of FDCS and signing with a FD had positive impact on utilization of primary health care services in the at community level. Also, individuals with chronic disease tend to use FDCS. More interventions to improve awareness of FDCS can help to increase the utilization of primary health care services.

\section{Introduction}

With the development of adequate controls for infectious diseases, such as vaccination and antibiotic use, China has experienced an epidemiological transition, shifting the focus from infectious diseases to noncommunicable diseases (NCDs) like diabetes and hypertension. For example, the prevalence of hypertension increased from $18 \%$ in 2002 to $25.2 \%$ in 2012 (1). Compared to treatments, prevention of NCDs at the community level has become more important. Even though the primary health care system was introduced to China in the 1980s, no attention was paid to establishing effective primary health care institutions, such as training primary health-care doctors and constructing insurance reimbursement systems. Due to the economic reforms in the early 1980s, the Chinese government reduced healthcare expenses and the market-oriented health care reforms caused an increased concentration of health care resources at higher-level care facilities, such as university-affiliated hospitals $(2,3)$. The neglect of the government's primary health care system caused discontent among the public (4). Thus, the Chinese government have been trying to strengthen the ability of health care services in primary health care system within recent years.

Community health care centers (CHCs) and community health care stations (CHSs) are the leading health care providers in the primary health care system and reform in urban China. Primary health care services provided by $\mathrm{CHCs}$ include essential public health services, such as immunization, and generalist clinical care, such as general physician (GP) health care services (2). One of the most critical healthcare reform policies was increasing subsidies to primary health care institutions from \$2.8 billion in 2008 to $\$ 20.3$ billion in 2015 (2). 
The number of CHCs increased from 24,000 to 35,000 in 2018 (1). The policy implementation of primary health care aims to provide affordable and accessible health care services.

With the implementation of primary health care, the concept of primary physicians was introduced in Chinese society. Before 2009, primary physicians included village doctors and "barefoot doctors" $(2,5)$. After 2009, the Chinese government began to identify physicians in primary health care centers, such as $\mathrm{CHCs}$, to play the gatekeeper's role. In 2011, the central government proposed the Guiding Opinions on Establishing a General Practitioner (GP) System to standardize the primary health care workforce (6). This guideline restricted the establishment of the GP system by training system and qualification exam. Family doctor contract services (FDCS) was published in 2016 (7). The GPs serve as family physicians (FPs) in the community and provide FDCS. FDCS are the extension of health care services based on voluntary signing contract with residents in the communities, providing consistent health care services, and building stable relationship with community's residents and. To use FDCS, individuals need to sign the service contract with a FD (8).

With the publication of "Healthy China 2030" in 2016, it suggested that FDCS should play an essential role in achieving the goal of Healthy China 2030, which is to improve population health by changing lifestyles, preventing and managing chronic diseases $(9,10)$. As FDs were designed to serve community residents in $\mathrm{CHCs}$, FDs should become a bridge and referral mechanism between secondary or tertiary hospitals, managers of NCD prevention, and community health monitors $(2,9)$. The adoption of the FD policy improved utilization of primary health care services for community residents. In 2016, the National Medical Reform Office released a new document to increase community residents' willingness to visit and sign with FDs (11). This document proposed the primary serving population as the elderly, pregnant women, children, individuals with disabilities, and those with hypertension, diabetes, and other chronic diseases. According to the document, in 2017, the FD signing rate should have reached $30 \%$ for the general population and $60 \%$ for the primary serving population. The document also suggested by 2020 , the coverage of the general population should reach $100 \%$ of the population. Meanwhile, FDs should hold a stable contract relationship with residents in the communities.

According to previous studies, FDs can effectively save health care resources and manage patients with chronic diseases $(12,13)$. However, the willingness to visit and sign with FDs have not been well identified. Recently, more researchers have started exploring the utilization of FDCS. Studies have indicated that the awareness of FDCS and the FD signing rate were low $(14,15)$. Other research also suggested that NCDs patients were more likely to have FDCS (16). Based on previous researches concentrated on estimating the awareness of FDCS, willingness to sign FD, and satisfaction of FDCS, it was found that FDCS provided efficient health care services in the communities $(9,15,17)$. Most of these studies or surveys were conducted in Shanghai $(9,12,16-18)$, Guangzhou (19), and Zhenjiang province (15). There is an urgent need to examine the primary health care system, FDs, and FDCS in other communities and cities within China. Shenzhen, a southeastern metropolis located in Guangdong, China, and China's first Special Economic Zone (SEZ), where it attracts millions of migrants to work, live, and study. Thus, the importance of studying the awareness of FDCS, willingness to sign with FD, and utilization of primary health care services in Shenzhen is profound.

Andersen's Behavioral Model of Health Services Use is widely used in health care utilization research (20). This model provides a conceptual framework for understanding different dimensions of access to medical care and individuals' decision to use health care services. The determinants of utilization of primary health care 
vary across different geographical locations, population construction, and socio-economic settings $(2,3,9,12$, $14-16,18,19,21-23)$. The relationship between FDs and CHC utilization is not well identified. Also, the relationship between chronic disease and FDs has not been well understood. We adapted Andersen's Behavioral Model of Health Services Use to identify factors that potentially facilitate FDs' impact on $\mathrm{CHC}$ utilization and the impact of chronic diseases on FD utilization. According to the model, the utilization of CHCs is determined by three factors, predisposing, enabling, and needs. Predisposing factors are demographic characteristics, such as age, gender, education, and marital status. Enabling factors can be defined as insurance status, such as the availability of insurance. This research estimated two perspectives of needs. First, the awareness of FD and the likelihood of signing with a FD were used to estimate the utilization of $\mathrm{CHCs}$. Second, the status of chronic disease was used to estimate the awareness of FD and the likelihood of signing with a FD $(12,24)$.

Compared to Shanghai, Shenzhen has a shorter history of FDCS. Shanghai was the first city to pilot FDCS in 2013 (9). Shenzhen published the FD guideline and policy at the end of 2017, and established the FDCS in 2018 (8). The function of FDs in Shenzhen is the same as in other cities. With the development and transformation of the economy, Shenzhen attracts millions of rural laborers and non-permanent migrants to work in the city (22). In 2018, the permanent year-end population was 13.02 million in Shenzhen, and 24.24 million in Shanghai. However, the year-end registered population was 4.55 million in Shenzhen, and 14.63 million in Shanghai suggested that the migration rate was $65 \%$ in Shenzhen, which was higher than $40 \%$ in Shanghai $(25,26)$. Compared to Shanghai, the policy implementation of FDCS may have a different impact on the utilization of health care services in CHCs. To the authors' knowledge, limited research has conducted to estimate the impact of awareness of FDCD on probability of using $\mathrm{CHCs}$, and the impact of signing with a FD on probability of utilizing community based CHCs in Shenzhen. Furthermore, limited research has been done to estimate the impact of having any chronic diseases on awareness of the FDCS and signing with a FD.

The purpose of this study is to identify effects, if any, of the awareness of FDCS on the probability of healthcare-seeking behavior change, and of having signed with a FD on healthcare-seeking behavior. We hypothesized that being aware of the FD services increases the probability for community residents to select $\mathrm{CHCs}$ as their first choice of health care institute. We also hypothesized that signing with a FD increases community residents' probability of choosing $\mathrm{CHCs}$ as their preferred health care institute. Furthermore, we also hypothesized that individuals with chronic disease are more likely to be aware of FDCS and have signed with a FD.

\section{Methods}

\section{Data and study population}

Data were collected as part of the China Community Diagnosis Survey. The China Community Diagnosis Survey is a community health diagnosis program operated by the Centers for Disease Control and Prevention (CDC) of Shenzhen to track community health care needs in the Dongmen community within the Luohu district of Shenzhen, China, in 2019. The Community Diagnosis Survey is conducted by the CDC of Shenzhen every three years. Objective of the survey is to identify community's health care problems, and to improve evidence-based health care promotion. Participants were community residents who lived in the districts for 
more than 6 months. Multistage cluster sampling was used to identify the sample of 1,205 residents. Seven sub-communities in the Dongmen community, Luohu district were selected. Twenty-five clusters were randomly selected based on the community grid from these seven sub-communities. A total of 925 households were interviewed face-to-face by trained investigators. Participants were Shenzhen residents who agreed to participate. If the selected household was not available, a nearby neighbor was chosen as a replacement, but the replacement rate was strictly controlled within $5 \%$ of the total sample. A total of 2,086 individuals were interviewed and 1,205 residents between 18 and 59 years old were included in the study. All participants provided a written informed consent before participated in the survey. This secondary data analysis was approved by the IRB of the corresponding author.

\section{Variables}

The first question aims to identify whether the awareness of FDCS and signing with a FD would change community residents' health seeking behaviors. The outcome variables included the willingness to use $\mathrm{CHCs}$, which was estimated by the question "Which medical institutions do you frequently visit?" The response options included 1) private clinics, 2) CHCs, 3) secondary hospitals, or 4) tertiary hospitals. The response was recoded as binary variable into 1) CHCs or 2) Others. The independent variable was measured with the question of "Have you ever heard about FDCS?" The response options included 1) Yes or 2) No. The other independent variable was measured with the question, “Did you sign a service contract with a FD?". The response options included 1) Yes and 2) No.

The second question aim to identify the efficiency of FDCS for community residents with chronic disease. The efficiency of FDCS were estimated by the awareness of the FDCS and signed with a FD. For the second part of estimation, the independent variable was measured with the following questions: "Do you have hypertension?" (Yes or No); “Do you have diabetes?” (Yes or No); “Do you have any following disease? Stroke, chronic bronchitis, chronic hepatitis $B$, hyperlipemia, cataract, osteoporosis, prostatauxe, cervical spondylopathy, chronic enteritis, chronic rhinitis, renal calculus, psychosis, benign tumors, malignant tumors, coronary disease, asthma, or other chronic disease?" (Yes or No). The response was recoded as a binary variable including: 1) Yes, have chronic diseases, or 2) No, do not have any chronic diseases.

Socio-demographic information included: hukou status (Shenzhen hukou, non-Shenzhen hukou), age (18-29, 30-39, 40-49, 50-59), gender (male, female), marital status (Yes or No), education (no education or primary school, middle school, high school, or professional college or university), employment status (Yes or No), and insurance status (Yes or No).

\section{Statistical analysis}

The relationships between health care seeking behavior and the awareness of FDCD and likelihood of signing with a FD were estimated using chi-square test and linear probability models (LPM) via ordinary least squares (OLS), respectively. LPM coefficients, reported as percentage change, directly estimated the change in magnitude and direction of probabilities of health care seeking behavior. Differences in the efficiency of FDCS, represented as percentage changes, between having and not having any chronic disease were examined using LPM via OLS. All regression models were adjusted for socio-demographic information, including age, marital 
status, education, gender, and employment status. Bootstrapped standard error was estimated. Alpha levels were set at 0.05. All analyses were conducted using Stata/SE 16 (StataCorp LP, College Station, TX). Alpha levels were set at 0.05 .

\section{Results}

A total of 1,205 adults were included in the analysis. Table 1 presents the characteristics of the sample by of awareness of FDCS and signed with a FD. About $26.8 \%$ of participants knew about FDCS, but only $5.2 \%$ of participants confirmed that they had signed with a FD. About $55.7 \%$ of participants who knew about FDCS select $\mathrm{CHCs}$ as their most frequently visited health care institutions, compared to those who did not know about the FDCS $(p<.01)$. About $61.9 \%$ of participants who signed with a FD chose CHCs as their most frequent visited health care institutions, compared to those who did not sign with a FD $(p<.01)$. Nearly $62.4 \%$ of participants were migrants, about $25.1 \%$ of them knew about FDCS, which is lower compared to local residents $(p<.01)$. The rate of signing with a FD was also lower among migrants, at only $22.2 \%$.

The characteristics of the sample by chronic diseases status are presented in Table 2 . The prevalence of having at least one chronic disease among community participants is $20 \%$. Among participants with chronic diseases, $35.2 \%$ of participants knew about FDCS and $11.9 \%$ of them had signed with a FD, compared to $64.8 \%$ of participants did not know the services and $88.1 \%$ of them did not sign with a FD $(p<.01)$.

The impact of FDCS' awareness and signing with a FD on utilization of CHCs was estimated by LPM shown in Table 3, respectively. Our analysis found that individuals knowing the FDCS were 18.5 percentage points ( $p<$ .001) more likely to choose CHCs as their first choice of health care institutes compared to those that do not know about the FDCS. The results of LPM found that participants who signed with a FD were 21.2 percentage points $(p<.01)$ more likely to choose CHCs as their first choice of health care institutes compared to those do not know FDCS. Among participants who were aware of FD service or signed with a FD, insurance coverage increased the likelihood of using CHCs by 18.5 percentage points and 20.4 percentage points, respectively.

The results of being aware of FDCS and signing with a FD by chronic disease status are presented in Table 4. The results indicated that participants with chronic diseases were 7.5 percentage points $(p<.05)$ more likely to be aware of FDCS, compared to participants without any chronic disease. The results also indicated that participants with chronic diseases were 6.5 percentage points $(p<.01)$ more likely to sign with a FD, compared to participants with no chronic disease. Additionally, the awareness of FDCS increased with education level; in particular, individuals with professional college or university education were 22.2 percentage points $(p<.001)$ more likely to know about FDCS, compared to those with no education or a primary school education. However, no statistically significant relationship was found between having a professional college or university education and signing with a FD ( $p>.05)$.

\section{Discussion}

The results of this study indicate a positive impact of the FD policy implementation in the communities in Shenzhen. Individuals were more likely to use CHCs as their first choice of health care institutes, if aware about FDCS or sign with a FD. Even though individuals in the communities are free to visit any type of medical 
institutions, they were still more willing to utilize CHCs compared to secondary or tertiary hospitals, which suggests that FDs have a positive effect among residents in the communities in utilizing CHCs. The establishment of long term relationships between FDs and communities' residents showed that individuals living in Shenzhen were able to accept the FDCS, which was consistent with the results from previous studies conducted in other cities $(9,15,18,19)$. The results imply that residents in this community were satisfied with FDCS. On the other hand, this also shows that there is still much room for improving the awareness rate about FDCS for residents in Shenzhen.

The results of this study also indicated a positive impact on the chronic disease population, who were more likely to have awareness of the FDCS and to have signed with a FD. Even though the signing rate was only $11.9 \%$ in the communities, the intention to sign with a FD was positive. One of the most important roles of FDs is chronic disease prevention and management. Previous studies have shown similar results that individuals with chronic diseases were more likely to sign with a FD and use FDCS $(14,21,23)$. In China, the FDCS package includes cost-saving and convenience provisions, such as chronic disease consultants, prescription drug refills, referral to secondary or tertiary hospitals, and health behavior interventions (27). Chronic disease prevention and prescription drug refills could be the most important benefits and driving forces for individuals with chronic diseases to sign with a FD.

Comparing to other cities, only $26.8 \%$ of participants in Shenzhen were aware of FD services, as well as only $5.2 \%$ of participants confirmed that they have signed with a FD in Shenzhen, 2019. Compared to the contract rate targets set by the government, which were $30 \%$ by 2017 and $100 \%$ by 2020 , the contracting rate of FD in Shenzhen communities was far from meeting the target contract rate (11). Compared to Shenzhen, the signed rate was much higher in other comparable cities, such as nearby cities of Guangzhou and Shanghai, with the contract rate ranging from $14.4-65.3 \%(9,12,18,19,21)$. The lower signed rate in Shenzhen could be explained by the high migration rate. According to the Shenzhen Statistical Yearbook, in 2019, only 35\% of the population in Shenzhen had Shenzhen houkou registration status. Most of the population in Shenzhen were not permanent residents of the city, especially migrant workers who often moved to other cities in pursuit of other opportunities. The high migration rate could contribute to the low contract rate with FDs, because the purpose and objective of FDs are to build up stable and consistent relationships with communities' residents regarding health-related behaviors. The short implementation time of FD policy may have led to a low signage rate of FD. The FD policy was introduced at the end of 2017, in November. Most of the population might not have had a chance to become familiar or be informed about the new policy of FDCS. Previous studies recruited study populations from CHCs, whereas our study population were recruited randomly from communities, which can better represent the health seeking behavior of the general population.

An interesting finding was the great impact of education on awareness of FDCS and likelihood of signing with a FD. Specifically, the probability of knowing about FDCS increased with the increasing of education levels. This could be largely explained by social media usage. Individuals with higher education level are more likely to absorb information from different mediums (28). Individuals with higher education levels have more resources to become aware of the FDCS and FD policy implementation. However, the probability of signing with a FD did not increase as the education level increased. This could be due to knowing about a certain policy often does not translate into action. Factors such as unwillingness and lack of motivation might hinder individuals' likelihood of signing with a FD. With an increased access to information and knowledge about 
FDCS and FD policy, it is possible that people might raise concerns about the quality of the service (29). This may explain the dilemma of the impact of education on awareness of FD and likelihood of signing with a FD.

Some suggestions can be made based on the results of this study. Even though the FD policy was implemented for a short period in Shenzhen, there is a need to further increase awareness of FDs and likelihood of signing with a FD. One important concept is the social determinants of health, which indicated individuals should be able to believe that community as a unit of health promotion projects $(30,31)$. FDs should have the availability and the capacity to serve as health promoters in the communities. To serve as health promoters, FDs should play the following roles: 1) building relationships with local community members, 2) increasing personal medical skills, and 3) increasing the acknowledgement of health promotion role of CHCs. The key concept is to build trust between FDs and community residents through providing home health care services to the community residents first. FDs cannot simply stay in the CHCs and wait for patients, instead, they should initiate contact with community residents. They can provide drop-in visits for some residents, such as individuals with chronic disease, individuals with disability, and the elderly. Social interaction is important to attract individuals to sign a service contract with a FD. Also, it is important to build up a consistent training environment. The distrust of primary care was mainly caused by the health care quality provided by GPs (2). FDs should be trained consistently either via self-training or government-provided health care training. The impact of the FD system will increase with the constant development of FDs. To expand the publicity of FD, social media should be used to advertise FDCS, such as using Weibo, Wechat, television, and newspaper. Diverse methods of health education and advertisements should be implemented to promote the efficiency and advantages of FDCS.

This study has some limitations. This study was a cross-sectional study with the focus of one district in Shenzhen. The data was collected through a self-reporting survey, self-reporting bias may exist. Also, this survey was not targeting on the primary health care in the community. It might not be able to capture all perspectives of FDSC and FD in Shenzhen. Lastly, this study only concentrated in Dongmen communities, Luohu District, which can limit the generalizability of the study. To fully investigate the contracted rate in Shenzhen, further studies need to be conducted on a larger scale.

\section{Conclusion}

Even though the rate of FD awareness and rate of signing with a FD were relatively low in Luohu, Shenzhen, they both have positive impacts on the utilization of CHCs. Populations with chronic diseases were more likely to know about FDs and to sign with a FD. The results of this study confirm the positive impact of FDs on health care seeking behavior change, aligned with previous studies. With the environment of policy implementation in primary health care, populations were gradually transforming their health care seeking behaviors. Community residents were more willing to utilize primary health care institutions, such as $\mathrm{CHCs}$, as their first choice of health care institutes. Such transformation in health care seeking behaviors was consistent with the direction of the FD policy implementation. It is also important for local government and society to make joint efforts to improve the medical services in the primary health care system, such as expanding public acceptance, improving health care quality for primary health care, and building up trust between community residents and FDs. 


\section{Abbreviations}

CHCs: Community health care centers

CHSs: Community health care stations

CDC: Centers for Disease Control and Prevention

FDCS: Family doctor contracted services

FD: Family doctor

FPs: Family physicians

GP: General physician

LPM: Linear probability models

NCDs: Non-communicable diseases

OLS: Ordinary least squares

SEZ: Special Economic Zone

\section{Declarations}

\section{Ethics approval and consent to participate}

All participants provided a written informed consent before participated in the survey collected by the Shenzhen Luohu Disease Prevention and Control Center. All methods associated with the survey and data analyses in this study were in accordance with the relevant guidelines and regulations. This secondary data analysis was reviewed by the Oregon State University's IRB and determined the study did not involve human subjects under the regulations set forth by the Department of Health and Human Services 45 CFR 46.

\section{Consent for publication}

Not applicable.

\section{Availability of data and materials}

The datasets generated during the current study are not publicly available due to the ownership belong of this dataset belong to Shenzhen Luohu Disease Prevention and Control Center, Shenzhen, Guangdong, China. The datasets used and/or analyzed during the current study available from the Shenzhen Luohu Disease Prevention and Control Center, Shenzhen, Guangdong, China on reasonable request.

\section{Competing interest}

The authors declare that they have no conflict of interest. 


\section{Funding}

There was no funding utilized for this research.

\section{Authors' contributions}

LS conceived the paper. QMZ led the systematic review and data analysis under LS direction. TTP and WL participated in the systematic review and data analysis. LS and QMZ wrote this paper. TTP and WL reviewed the paper. All authors read and approved the final manuscript.

\section{Acknowledgements}

We are grateful to the Shenzhen Luohu Disease Prevention and Control Center, Shenzhen, Guangdong, China for providing us the raw data from the Luohu Community Diagnosis Program.

\section{References}

1. National Bureau of Statistics. China Statistical Yearbook 20192019 [Available from: http://www.stats.gov.cn/tjsj/ndsj/2019/indexch.htm.

2. Li X, Lu J, Hu S, Cheng K, De Maeseneer J, Meng Q, et al. The primary health-care system in China. The Lancet. 2017;390(10112):2584-94.

3. Zhao Y, Lin J, Qiu Y, Yang Q, Wang X, Shang X, et al. Demand and signing of general practitioner contract service among the urban elderly: a population-based analysis in Zhejiang Province, China. International Journal of Environmental Research and Public Health. 2017;14(4):356.

4. Wu D, Lam TP. At a crossroads: Family medicine education in China. Academic Medicine. 2017;92(2):185-91.

5. Zhang D, Unschuld PU. China's barefoot doctor: past, present, and future. The Lancet. 2008;372(9653):1865-7.

6. The Central People's Government of the People's Republic of China. Guiding Opinions on Establishing a General Practitioner System 2011 [Available from: http://www.gov.cn/zhengce/content/201107/06/content_6123.htm.

7. The Central People's Government of the People's Republic of China. Notice on Issuing Guidance Opinions on Promoting Family Doctor Contract Services 2016 [Available from: http://www.nhc.gov.cn/tigs/s3577/201606/e3e7d2670a8b4163b1fe8e409c7887af.shtml.

8. Shenzhen Government. Shenzhen Family Doctor Service Management Methods 2017 [Available from: http://www.sz.gov.cn/zfgb/2017/gb1029/content/post_4945557.html.

9. Huang J, Liu S, He R, Fang S, Lu W, Wu J, et al. Factors associated with residents' contract behavior with family doctors in community health service centers: A longitudinal survey from China. PLoS One. 2018;13(11):e0208200.

10. Wei L. Health sector, the next big industry. chinadaily. 2016.

11. National Medical Reform Office. Notice on Promoting Guidance for Family Doctor Contracted Services 2016 [Available from: 
http://www.mohrss.gov.cn/SYrlzyhshbzb/shehuibaozhang/zcwj/yiliao/201606/t20160615_241854.html.

12. Huang J, Zhang T, Wang L, Guo D, Liu S, Lu W, et al. The effect of family doctor-contracted services on noncommunicable disease self-management in Shanghai, China. The International journal of health planning and management. 2019;34(3):935-46.

13. Lorig KR, Sobel DS, Stewart AL, Brown Jr BW, Bandura A, Ritter P, et al. Evidence suggesting that a chronic disease self-management program can improve health status while reducing hospitalization: a randomized trial. Medical care. 1999:5-14.

14. Zhang Yh, Zhang Th, Wang Zf. Status of Signing on Family Doctor Service for Residents in Desheng Area of Beijing and Its Influencing Factors (Chinese). Chinese General Practice. 2013;16(11).

15. Shang X, Huang Y, Li Be, Yang Q, Zhao Y, Wang W, et al. Residents' Awareness of Family Doctor Contract Services, Status of Contract with a Family Doctor, and Contract Service Needs in Zhejiang Province, China: A Cross-Sectional Study. International journal of environmental research and public health. 2019;16(18):3312.

16. Huang J, Lu W, Wang L, Zhang T, Liu C, Liu S, et al. A preliminary effect analysis of family doctor and medical insurance payment coordination reform in Changning District of Shanghai, China. BMC family practice. 2019;20(1):60.

17. Xie C-y, Hu S-I, He J-j. Thoughts and Suggestions of Family Doctor Services Based on Field Observation. Chinese General Practice. 2012;2012(31):11.

18. Yuan L, Zhou C, Jiang P, Xu L, Zhang Q, Xu B. Community residents' demand for family physician service in Changning Districe, Shanghai (Chinese). Chinese General Practice. 2014;17(32):3860-4.

19. Liu Z, Tan Y, Liang H, Gu Y, Wang X, Hao Y, et al. Factors Influencing Residents' Willingness to Contract With General Practitioners in Guangzhou, China, During the GP Policy Trial Phase: A Cross-Sectional Study Based on Andersen's Behavioral Model of Health Services Use. INQUIRY: The Journal of Health Care Organization, Provision, and Financing. 2019;56:0046958019845484.

20. Andersen RM, Davidson PL, Baumeister SE. Improving access to care. Changing the US health care system: key issues in health services policy and management San Francisco: Jossey-Bass. 2014;36(3):33-69.

21. Huang J, Liang H, Zhang W, Zhang J, Wang c, Chen X, et al. Influencing factor analysis of signing a contract with a family doctor among residents in Hongkou district (Chinese). Chinese General Practice. 2019;22(6):687-91.

22. Mou J, Cheng J, Zhang D, Jiang H, Lin L, Griffiths SM. Health care utilisation amongst Shenzhen migrant workers: does being insured make a difference? BMC health services research. 2009;9(1):214.

23. Wu J, Xu L, Lu M, Hu Y, Shen A, Shen J, et al. Demand of residents in Chengjiaqiao community for family physician services (Chinese). Chinese General Practice. 2012;15(16):1809-11.

24. Huang K, Song YT, He YH, Feng XL. Health system strengthening and hypertension management in China. Global health research and policy. 2016;1(1):13.

25. Municipality SBoS. Shenzhen Statistical Yearbook 2019 [Available from: http://tjj.sz.gov.cn/attachment/0/695/695422/7971762.pdf. 
26. Statistics Bureau of Shanghai Municipality. Shanghai Statistical Yearbook 20192019 [Available from: http://tjj.sh.gov.cn/tjnj/20200427/4aa08fba106d45fda6cb39817d961c98.html.

27. The State Council of the People's Republic of China. Guidance on promoting general practitioners' contract service 2016 [Available from: http://www.nhc.gov.cn/cms-search/xxgk/getManuscriptXxgk.htm? id=e3e7d2670a8b4163b1fe8e409c7887af.

28. Perrin A. Social media usage. Pew research center. 2015:52-68.

29. Tucker JD, Wong B, Nie J-B, Kleinman A. Rebuilding patient-physician trust in China. The Lancet. 2016;388(10046):755.

30. Jung M, Rhee HS. Determinants of community capacity influencing residents' health status in Seoul, South Korea. Asia Pacific Journal of Public Health. 2013;25(2):199-208.

31. Sampson RJ. Neighborhood-level context and health: lessons from sociology: Nueva York, Oxford University Press; 2003.

\section{Tables}


Table 1. Characteristics for health care seeking behavior (willingness to use CHCs as first choice) by awareness of the FDCS and signed with a FD in Dongmen community, Luohu district, Shenzhen, China

\begin{tabular}{|c|c|c|c|c|c|}
\hline Variable & Aware & of FDCS & P- & Signed wi & a FD \\
\hline & $\begin{array}{l}\text { No, } n \\
(\%)\end{array}$ & $\begin{array}{l}\text { Yes, n } \\
(\%)\end{array}$ & & No, n (\%) & $\begin{array}{l}\text { Yes, n } \\
(\%)\end{array}$ \\
\hline
\end{tabular}

Health care seeking behavior (willingness

to use $\mathrm{CHCs}$ )

$\begin{array}{lllllll}\text { Others } & 548 & 143 & <0.01 & 667 & 24 & <0.01 \\ & (62.13) & (44.27) & & (58.41) & (38.1) & \\ \text { CHCs } & 334 & 180 & & 475 & 39 & \\ & (37.87) & (55.73) & & (41.59) & (61.9) & \end{array}$

Hukou status

$\begin{array}{lllllll}\text { Locals (Shenzhen hukou) } & 281 & 172 & <0.01 & 404 & 49 & <0.01 \\ & (31.86) & (53.25) & & (35.38) & (77.78) & \\ \text { Migrants (non-Shenzhen hukou) } & 601 & 151 & & 738 & 14 \\ & (68.14) & (46.75) & & (64.62) & (22.22) & \end{array}$

Age

$18-29$

$\begin{array}{llllll}218 & 51 & <0.01 & 262 & 7 & <0.01 \\ (24.72) & (15.79) & & (22.94) & (11.11) & \\ 253 & 121 & & 356 & 18 & \\ (28.68) & (37.46) & & (31.17) & (28.57) & \\ 235 & 85 & & 305 & 15 \\ (26.64) & (26.32) & & (26.71) & (23.81) & \\ 176 & 66 & & 219 & 23 \\ (19.95) & (20.43) & (19.18) & (36.51) & \end{array}$

Gender

Male

\begin{tabular}{llllll|}
414 & 134 & 0.09 & 522 & 26 & 0.49 \\
$(46.94)$ & $(41.49)$ & & $(45.71)$ & $(41.27)$ & \\
468 & 189 & & 620 & 37 & \\
$(53.06)$ & $(58.51)$ & & $(54.29)$ & $(58.73)$ & \\
\hline
\end{tabular}

Marriage

$\begin{array}{lllllll}\text { No } & 234 & 48 & <0.01 & 275 & 7 & 0.02 \\ & (26.53) & (14.86) & & (24.08) & (11.11) & \\ \text { Yes } & 648 & 275 & & 867 & 56 & \\ & (73.47) & (85.14) & & (75.92) & (88.89) & \end{array}$

Education

No education or primary school

Middle school

$\begin{array}{llllll}131 & 17 & <0.01 & 147 & 1 & <0.01 \\ (14.85) & (5.26) & & (12.87) & (1.59) & \\ 261 & 64 & & 314 & 11\end{array}$

Page 13/20 


\begin{tabular}{|c|c|c|c|c|c|c|}
\hline & (29.59) & (19.81) & & (27.50) & (17.46) & \\
\hline High school & $\begin{array}{l}245 \\
(27.78)\end{array}$ & $\begin{array}{l}99 \\
(30.65)\end{array}$ & & $\begin{array}{l}321 \\
(28.11)\end{array}$ & $\begin{array}{l}23 \\
(36.51)\end{array}$ & \\
\hline Professional college or university & $\begin{array}{l}245 \\
(27.78)\end{array}$ & $\begin{array}{l}143 \\
(44.27)\end{array}$ & & $\begin{array}{l}360 \\
(31.52)\end{array}$ & $\begin{array}{l}28 \\
(44.44)\end{array}$ & \\
\hline \multicolumn{7}{|l|}{ Employment Status } \\
\hline No & $\begin{array}{l}137 \\
(15.53)\end{array}$ & $\begin{array}{l}65 \\
(20.12)\end{array}$ & 0.06 & $\begin{array}{l}182 \\
(15.94)\end{array}$ & $\begin{array}{l}20 \\
(31.75)\end{array}$ & $<0.01$ \\
\hline Yes & $\begin{array}{l}745 \\
(84.47)\end{array}$ & $\begin{array}{l}258 \\
(79.88)\end{array}$ & & $\begin{array}{l}960 \\
(84.06)\end{array}$ & $\begin{array}{l}43 \\
(68.25)\end{array}$ & \\
\hline \multicolumn{7}{|l|}{ Insurance Status } \\
\hline No & $\begin{array}{l}94 \\
(10.66)\end{array}$ & $7(2.17)$ & $<0.01$ & $\begin{array}{l}101 \\
(8.84)\end{array}$ & $0(0)$ & 0.01 \\
\hline Yes & $\begin{array}{l}788 \\
(89.34)\end{array}$ & $\begin{array}{l}316 \\
(97.83)\end{array}$ & & $\begin{array}{l}1041 \\
(91.16)\end{array}$ & $\begin{array}{l}63 \\
(100)\end{array}$ & \\
\hline
\end{tabular}


Table 2. Characteristics for awareness of FDCS and singed with a FD by chronic disease status in Dongmen community, Luohu district, Shenzhen, China

Variable

Chronic disease x2 P-value

No, n (\%) Yes, n (\%)

Awareness of the FDCS

No

Yes

Signed with a FD

No

Yes

Hukou status

Locals (Shenzhen hukou)

Migrants (non-Shenzhen hukou)

Age

18-29

30-39

40-49

50-59

Gender

Male

Female

Marriage

No

Yes

Education

No education or primary school

Middle school

High school

Professional college or university

Employment Status

No

$729(75.23)$

$240(24.77)$

934 (96.39)

35 (3.61)

335 (34.57)

$634(65.43)$

118 (50.00)

$118(50.00)$

28 (11.86)

83 (35.17)

208 (88.14)

$<0.01$

(3.61)

(11.86)

$\sqrt{2}+2$

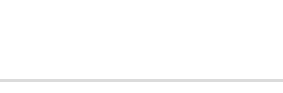


Insurance Status

$\begin{array}{llll}\text { No } & 84(8.67) & 17(7.20) & 0.47 \\ \text { Yes } & 885(91.33) & 219(92.8) & \end{array}$

Note. Data from Community Diagnosis Questionnaire, Luohu, Shenzhen (2019). FD: Family doctor; FDCS: family doctor contract services; CHCs: community health care centers. 
Table 3. Linear probability models of factors associated with health care seeking behavior by awareness of the FDCS and signed with a FD in Dongmen community, Luohu district, Shenzhen, China

Variable

Health care seeking behavior (willingness to use CHCs)

Percentage point change (bootstrapped SE\%)

Awareness of the FDCS

No

Yes

Signed with a FD

No

Yes

Hukou status

Locals (Shenzhen hukou)

Migrants (non-Shenzhen hukou)

Age

$18-29$

30-39

40-49

50-59

Gender

Male

Female

Marriage

No

Yes

Education

No education or primary school

Middle school

High school

Professional college or university

Employment Status

No

Ref.

Ref.

Ref.

Ref.

\section{$21.19 * *(6.73)$}

Ref.

$5.41(3.56)$

$5.61(3.72)$

Ref.

$4.37(4.62)$

$5.32(4.57)$

$6.67(4.49)$

$6.85(4.43)$

$2.57(5.64)$

$2.28(5.61)$

$1.23(3.07)$

$2.10(3.03)$

$3.36(4.09) \quad 5.13(4.09)$

$\begin{array}{ll}1.72(5.15) & 2.76(5.14) \\ -04.11(5.58) & -1.83(5.54) \\ -10.24(5.85) & -6.80(5.92)\end{array}$

$-6.80(5.92)$ 
Insurance Status

No Ref.

Yes

$18.54^{\star \star \star}(4.78)$

$20.39 * \star \star(4.86)$

Note. Data from Community Diagnosis Questionnaire, Luohu, Shenzhen (2019). FD: Family doctor; FDCS: family doctor contract services; CHCs: community health care centers. ${ }^{\star} p<0.05$, ${ }^{\star \star} p<0.01,{ }^{\star \star \star} p<0.001$. 
Table 4. Linear probability models of factors associated with awareness of the FDCS and signed with a FD by chronic disease status in Dongmen community, Luohu district, Shenzhen, China
Variable
Awareness of the FDCS
Signed with a FD
Percentage point change (bootstrapped SE\%)

Chronic disease

No Ref.

Yes

$7.47 *(3.12)$

$6.50 * \star(2.07)$

Hukou status

Locals (Shenzhen hukou)

Ref.

Migrants (non-Shenzhen hukou)

$-7.03^{*}(3.44)$

$-7.08^{\star \star \star}(1.78)$

Age

18-29

Ref.

30-39

$3.84(4.15)$

$-1.11(1.87)$

40-49

$-0.89(4.69)$

$-1.60(2.04)$

50-59

$0.55(5.19)$

$1.85(2.13)$

Gender

Male

Ref.

Female

$5.60 *(2.48)$

$0.78(1.30)$

Marriage

No

Ref.

Yes

$12.40^{\star \star \star}(3.74)$

$2.52(1.76)$

Education

No education or primary school

Ref.

Middle school

$9.71 * *(3.62)$

$3.57^{\star \star}(1.28)$

High school

$17.61^{\star \star \star}(4.06)$

$4.63^{\star \star}(1.59)$

Professional college or university

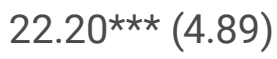

$3.19(1.91)$

Employment Status

No

Ref.

Yes

$-3.48(3.51)$

$-3.30(2.30)$

Insurance Status

No

Ref. 
Note. Data from Community Diagnosis Questionnaire, Luohu, Shenzhen (2019). ${ }^{*} p<0.05,{ }^{* *} p<0.01, * \star *$ $p<0.001$. FD: Family doctor; FDCS: family doctor contract services; $C H C s:$ community health care centers.

\section{Supplementary Files}

This is a list of supplementary files associated with this preprint. Click to download.

- Questionnaire.docx

- Questionnaire.docx 